\title{
Research of Wave Simulation Based on Nonlinear Constraint
}

\author{
Bingjie Jiao* \\ North China Electric Power University, Baoding 071000, China \\ *15803104698@163.com
}

\begin{abstract}
Nowadays, one of the most significant signal carriers, the radio wave of high frequency propagates in multi-hop path. We establish the foundation model of Sky-wave reflection in order to study the relationship between some factors (signal intensity, SNR (Signal to Noise Ratio), etc.) and propagating distance, and solve out the optimal multi-hop path. We assume that the value of projecting power of radio wave, the distance between radio wave source and receiver and times of hop are known and we establish the basic model of the calm ocean to determine the signal intensity and SNR at the target location.
\end{abstract}

Keywords: SNR; optimal multi-hop path.

\section{Introduction}

High frequency (HF), electromagnetic waves (radio waves) whose frequency is between 3 and 30 megahertz (MHz). It is also known as a very useful role in our daily life. Besides, the main users of the high frequency spectrum are not only military and governmental communication systems, but also many scientists who study on maritime stuffs.

HF plays a significant role in our lives. Furthermore, the dominant means of long distance communication in this band is skywave ("skip") propagation, in which radio waves directed at an angle into the sky reflect back to Earth from layers of ionized atoms in the ionosphere. While approximately $70 \%$ of the earth is ocean, we decide to focus on the reflections from ocean majorly. It is said that the roughness of the ocean determines the reflection of the electromagnetic wave. It is said that the energy of reflections off the calm ocean are slightly more than those reflections off the turbulent ocean from experiences.

Radio wave as a signal carries information. To measure the capability of radio wave transferring information, we introduce a physical quantity: SNR(Signal to Noise Ratio).SNR is the ratio of the strength of signal to the strength of atmospheric noise, it is an effective way to know if the signal is transmitted completely.

\section{The Circumstances on the Calm Ocean}

\subsection{Establish the Reflection Model on the Calm Ocean(RMCO)}

The task requires us to solve out the maximum number of hop before the SNR of radio wave is less than 10dB.SNR is determined by many factors. Hence, we set our target. And in order to solve out the problem, we also set some restrictions.

Target:

To solve out the maximum times of hop before SNR is less than $10 \mathrm{~dB}$ :

$\max n$

Restrictions:

$$
(S N R)_{L} \geq 10 d B
$$

To satisfy the requirement, the SNR of last hop is equal or greater than $10 \mathrm{~dB}$; 


$$
(S N R)_{L}=E_{t}-E_{n}
$$

$(S N R)_{L}$ equals to the difference between $E_{t}$ and $E_{n}$;

$$
E_{t}=137.2+20 \lg f+P_{t}-L_{b f}
$$

The intensity of the radio wave that receiver received is determined by $f, P_{t}, L_{b f}$

$$
E_{n}=-0.8379 f+24.3293
$$

The strength of atmospheric noise $E_{n}$ is proportional to negative $\mathrm{f}$

$$
200 \mathrm{~km} \leq \mathrm{D} \leq 2000 \mathrm{~km}
$$

The distance between two positions (the length along the great circle every one hop) D, because in the distance which above $200 \mathrm{~km}$ is suitable for the range of radio wave frequency in HF to transmit;

$$
3(\mathrm{MHz}) \leq f \leq \mathrm{MUF}(\mathrm{MHz})
$$

The frequency of radio wave should be restricted in $3 \mathrm{MHz} \sim \mathrm{MUF}(\mathrm{MHz})$

\subsection{Algorithm}

We apply the basic model to the problem and use MATLAB to draw some stereograms to solve the maximum number of hop. Since higher the frequency of the radio wave (the frequency range is HF), the more reflections off ionosphere. And we set $\mathrm{n}$ (the number of hop) and other parameters, then get the relationship between working frequency $(3 \mathrm{MHz} 30 \mathrm{MHz})$, SNR (signal-to-noise ratio) and distance(D).

Here is our algorithm as follows:

Initial conditions:

We assume the season is summer, which is of frequent thunderstorms.

We set $R_{12}=20 ; \mathrm{MUF}=30 \mathrm{MHz} ; P_{t}=100 \mathrm{w} ; \chi=10^{\circ} ; \mathrm{H}=110 \mathrm{~km}$;

The results as Fig.1:

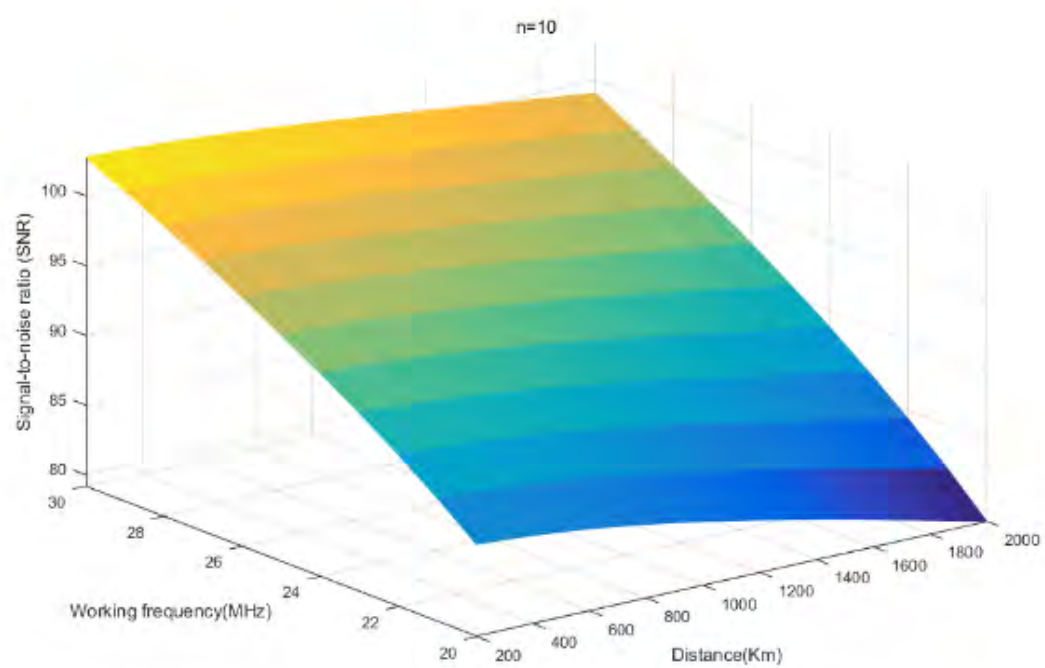

Figure 1. Results of the algorithm $(\mathrm{n}=10)$ 
The stereogram shows the relationship between SNR, working frequency and distance when $\mathrm{n}=10$.It is clearly that the wave frequency ranging from $3 \mathrm{MHz} 30 \mathrm{MHz}$ can take 10 hops before their SNR less than 10dB.To maximize the number of hops, we decide to increase the value of $\mathrm{n}$ (from 10 on)
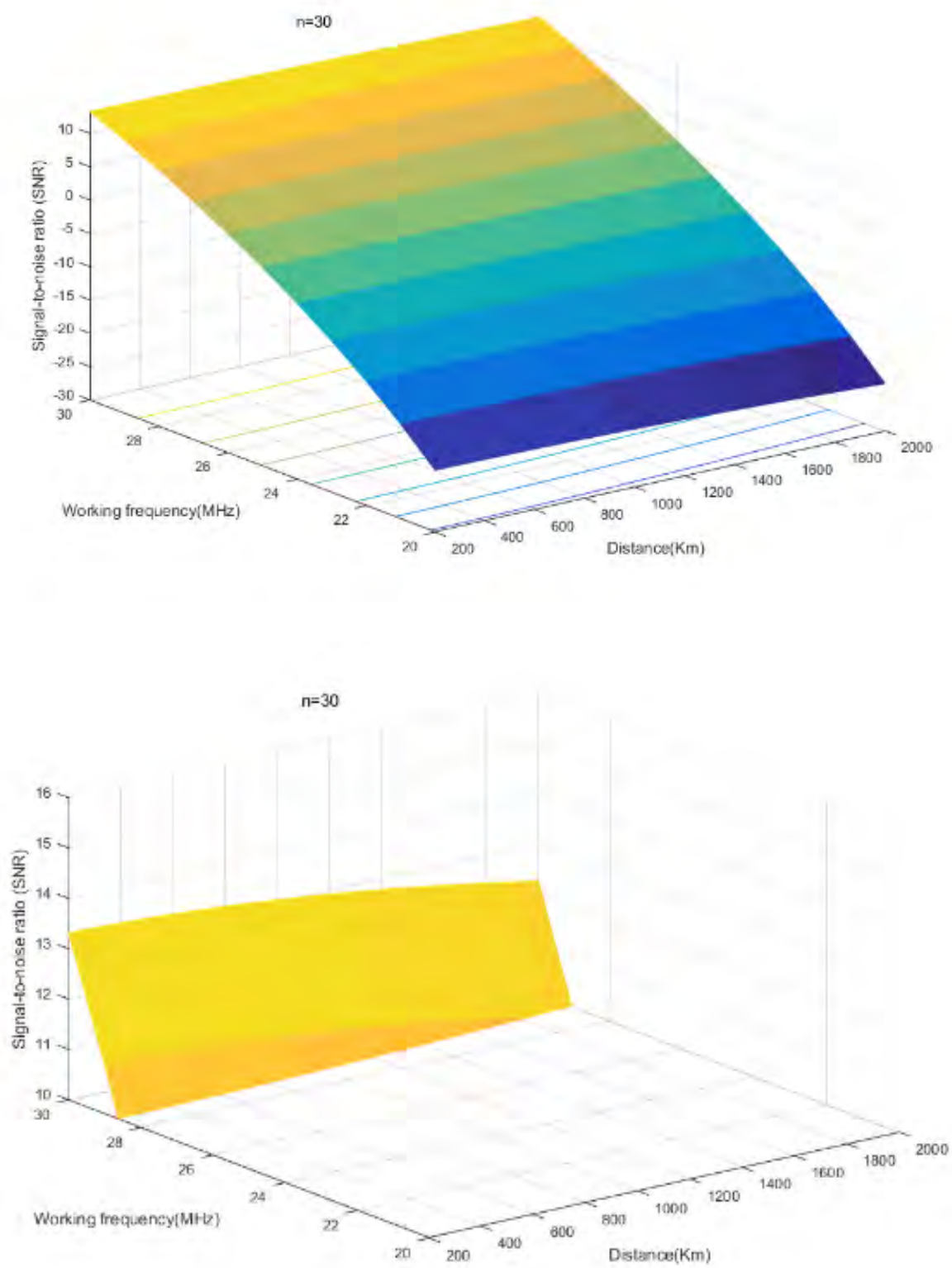

Figure 2. Results of the algorithm(n=30)

When we set $n=30$, and construct two stereograms as Fig.2. The figure on the left side shows the SNR of the radio wave frequency ranging from $3 \mathrm{MHz} 30 \mathrm{MHz}$.In the meantime, from the figure on the left side, the remain of the curve across by the 10dB-cross-section plane, we can see that only the radio wave whose frequency is around $28.5 \mathrm{MHz} 30 \mathrm{MHz}$, very close to the MUF $(30 \mathrm{MHz})$

When we set $n=31$, we wonder if $n=31$ can be the maximum number. Hence, we draw the stereogram when $n=31$. The following stereograms show the relationship between $n$, working frequency and distance when $n=31$. 

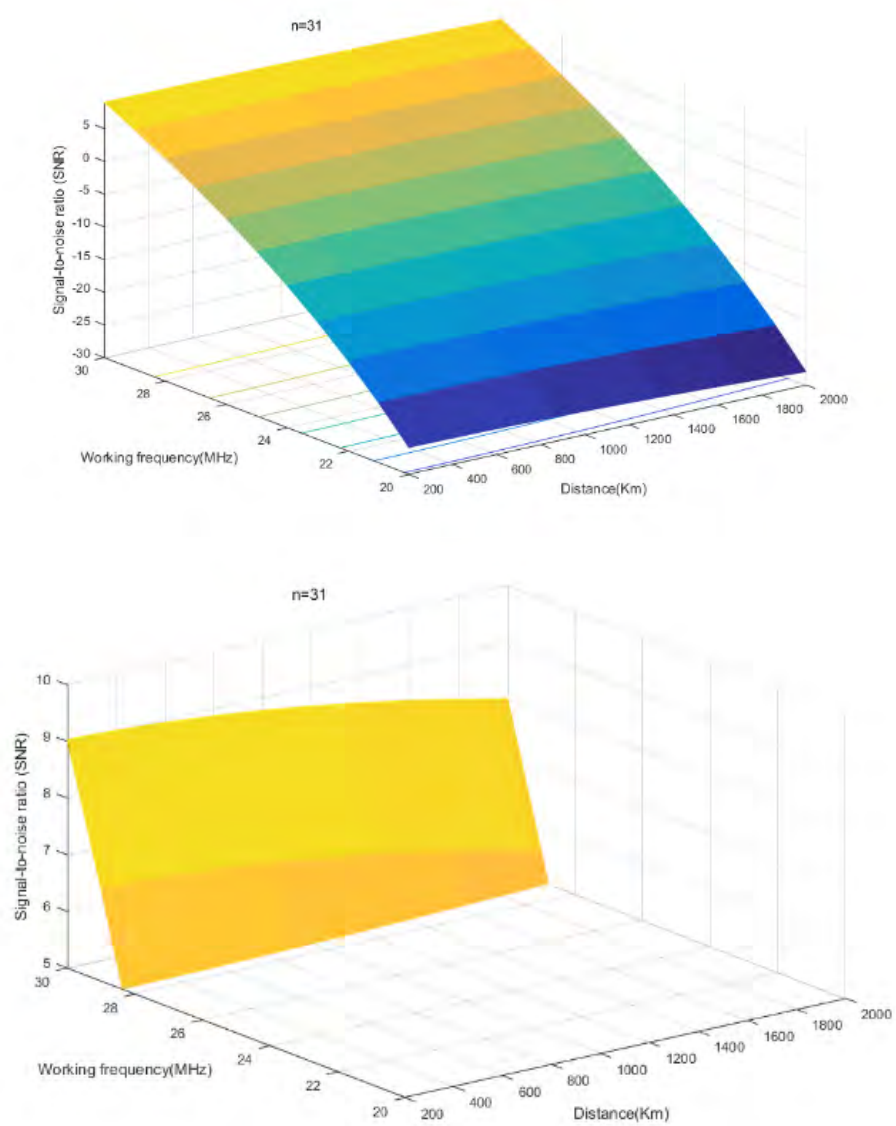

Figure 3. Results of the algorithm $(\mathrm{n}=31)$

It is clearly shown in Fig.3 that even the frequency of radio wave is very close to MUF, no radio wave whose SNR is above $10 \mathrm{~dB}$ when $\mathrm{n}$ equals to 31 .

At last we solve out the result: To satisfy the requirement the maximum times of hop is 30 .

\subsection{The First Reflection off the Ocean}

We apply the basic model and determine the intensity of the first reflection.

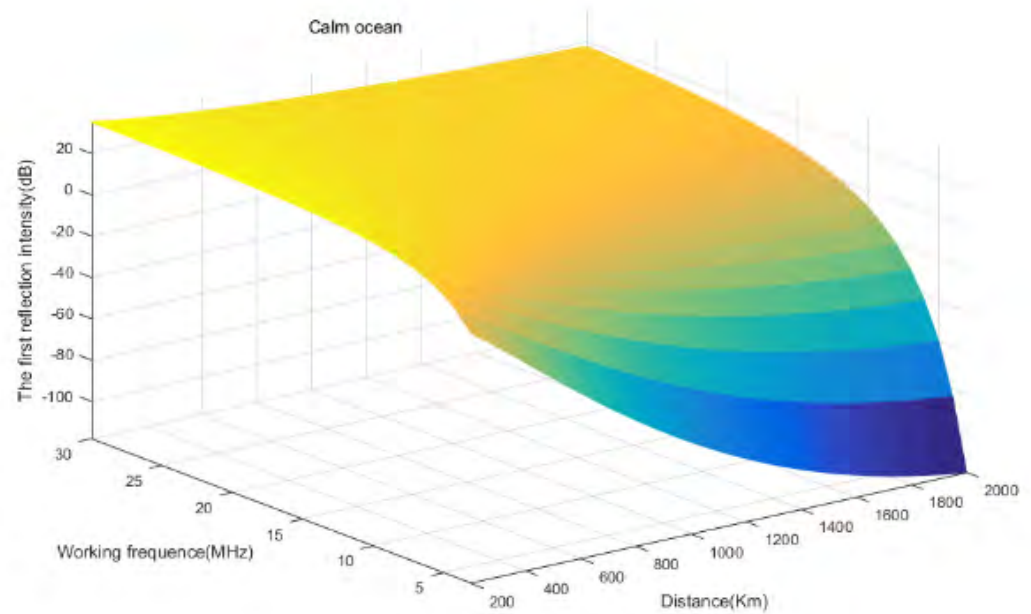

Figure 4. The first reflection intensity of the radio

Figure. 4 shows that the first reflection intensity of the radio wave which frequency ranging from $3 \mathrm{MHz} 30 \mathrm{MHz}$ at every position in the space. 


\section{Sensitivity Analysis}

In order to analyze the sensitivity, we fix this factors:

$\mathrm{f}=15 \mathrm{MHz}, \mathrm{D}=400 \mathrm{~km}, \mathrm{H}=110 \mathrm{~km}, \mathrm{DOT}=3, \mathrm{P}=0.1 \mathrm{~kW}, R_{12}=20, \chi=10^{\circ}$.

After fixing these factors, we draw a curve under a coordinate which x-axis is the number of hop while $y$-axis is the signal intensity. This curve shows that the signal intensity reduces with the increment of times of hop.

Therefore, we only change the factor that we study on and draw curves which change the studying factor, then observe the new curve's offset of the original one.

\subsection{Sensitivity to Solar Conditions}

We change solar conditions to explore the influence on our models.

\subsubsection{The Number of Sunspots $\left(R_{12}\right)$}

To explore the influence on models when we change the number of sunspots. We consider the number of sunspots only influence the energy loss of ionosphere. Therefore, the following figure shows the curves under the change of the number of sunspots.

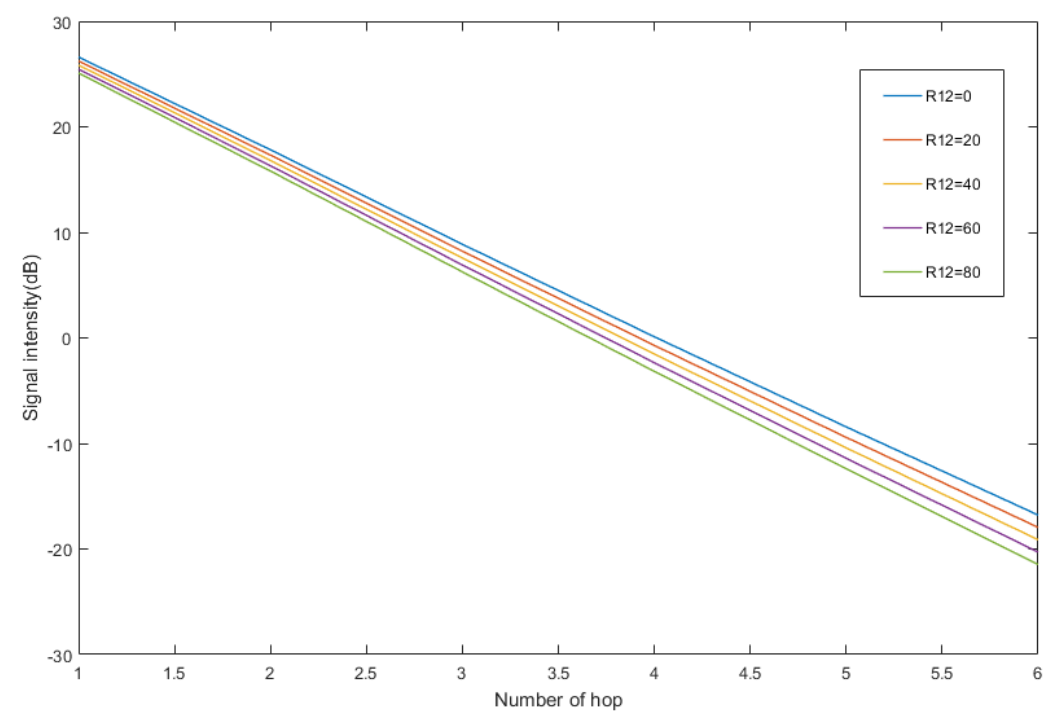

Figure 5. The curve of the number of sunspots

Fig. 5 shows the influence on the parameter of our models (signal intensity). Greater the number of sunspots, less the signal intensity. The difference between curves in the model increase with the increment of times of hop.

\subsubsection{The Solar Zenith Angle $(\chi)$}

We change the solar zenith angle and explore the influence on the model. Therefore, the figure below shows the relationship between the solar zenith angle and the signal intensity.

As shown in Fig.6, it is clearly that greater the solar zenith angle, stronger the signal intensity. Besides, there are significant disparities between these curves. 


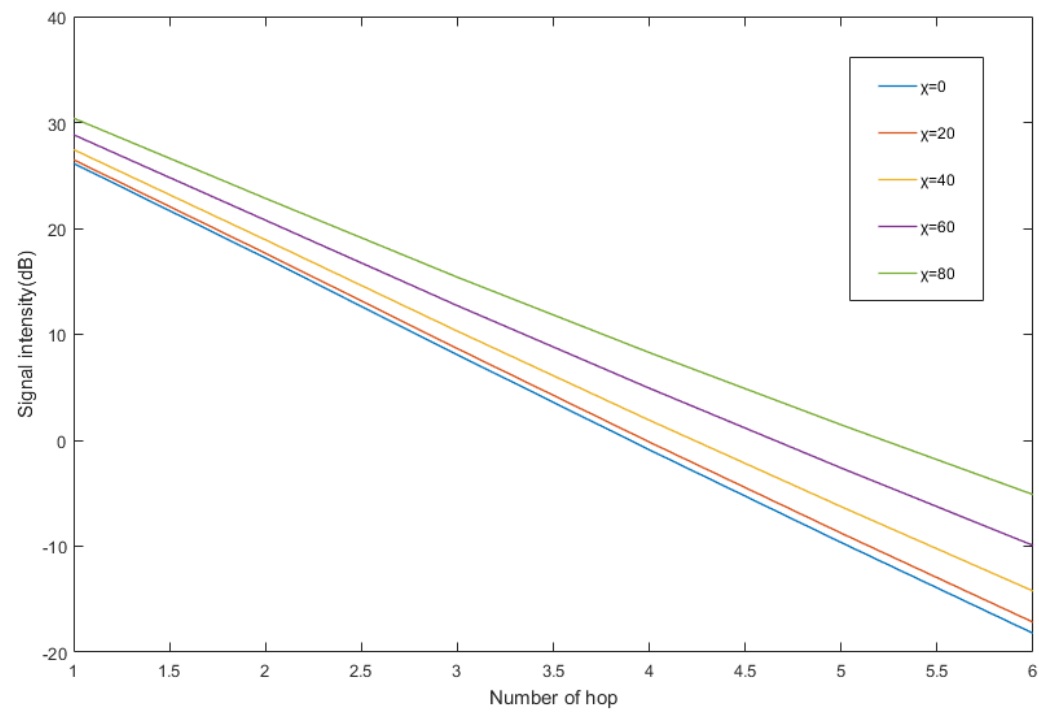

Figure 6. The relationship between solar zenith Angle and signal intensity

\section{Summary}

In this paper, we analyzed the reflection on the calm sea and Establish the Reflection Model on the Calm Ocean(RMCO). We specify our basic model to the circumstances on the calm ocean and draw some stereograms to solve out the maximum times of hop.

\section{References}

[1]. Academy D N, Dalian. Research on the Feasibility of Shortwave Communication at Sea under the Condition of Atmosphere Yawp[J]. Ship Electronic Engineering, 2009.

[2]. Guilin Y E. Calculation and Analysis of HF Communication Circuit at Sea[J]. Ship Electronic Engineering, 2017.

[3]. Wireless channel model simulation and hardware implementation of irregular terrain, Ong Hai.

[4]. Zhuang-bo, sun fang. Analysis of impact of different ground forms on short-wave communication [J]. China new communication, 2014 (9):92-92.

[5]. Dong Hai. Wireless channel model simulation and hardware implementation design of irregular terrain [D]. National defense science and technology university, 2009. 